\title{
Fenología de la floración del cafeto var. Catuaí Rojo en el municipio Caripe del estado Monagas, Venezuela
}

\author{
Phenology of the flowers bloom over var. Catuai Rojo in the Caripe \\ municipality of the state Monagas, Venezuela
}

\author{
Martín Coa Urbaez, Ramón Silva-Acuña ${ }^{2}$, Jesús Rafael Méndez Natera², Sol Mundarain Padilla ${ }^{2}$
}

\section{RESUMEN}

Con el objetivo de describir las fases fenológicas de floración del café empleando la escala ampliada BBCH se utilizó plantas de Coffea arabica L., var. Catuaí Rojo de 10 años de edad, sembradas a 1,0 x 1,5 m., a libre crecimiento bajo condiciones de sombrío, provisto por árboles de bucare (Eryhrina glauca Willd) a un distanciamiento de 14 x $14 \mathrm{~m}$. De forma aleatoria en el área experimental se seleccionaron 30 plantas de café y en su tercio medio se marcaron cuatro ramas plagiotrópicas, dos dirigidas en dirección este y dos en dirección oeste, para 120 ramas. Las observaciones se realizaron a intervalos de tres días durante el período febrero a mayo de 2008. De acuerdo con los resultados obtenidos se identificaron seis estadios fenológicos de la floración: 51-yemas hinchadas; 53 yemas visibles o botones florales indiferenciados; 57 yemas comprimidas o botones florales en desarrollo; 58 botones florales diferenciados o en latencia; 59 botones florales en preantesis y $>60$ botones florales en antesis o apertura floral. El período desde la aparición del hinchamiento de la axila floral hasta la floración tuvo una duración de 88,2 días. Se atribuye al estrés hídrico la responsabilidad acerca de la ocurrencia del estadio fenológico de inducción floral. La ruptura de la latencia se debe a las precipitaciones ocurridas al final de abril con un registro acumulado de 57,20 mm.

Palabras claves: Coffea arabica L., estadios fenológicos, floración.

\begin{abstract}
With the objective to describe the phenological stages of flowering of the coffee using the extended scale BBCH, used plants of Coffea arabica L. , var. Catuai Rojo 10 years of age, planted at $1.0 \times 1.5 \mathrm{~m}$., to free growth under conditions of bleak, provided by Bucare trees (Eryhrina glauca Willd) to a distance of $14 \times 14 \mathrm{~m}$. Randomly in the experimental area was 30 plants were selected and coffee and in its middle third is marked plagiotropicas four branches, two directed in this direction and two in a westerly direction, for a total of 120 branches. Observations were made at intervals of three days during the period February to May of 2008. In accordance with the results obtained were identified six phenological stages of flowering; 51-buds swollen, 53-buds floral buttons visible or undifferentiated, 57-buds pinched or buttons in floral development, 58-buttons differentiated or floral in latency, 59-buttons in floral anthesis and > 60-buttons in floral anthesis or opening. The period from the appearance of swelling of the axilla floral to flowering had duration of 88.2 days. Is attributed to water stress the responsibility on the occurrence of the phenological stage of floral induction. The breakdown of the latency is due to the rains that occurred at the end of the month of April with a cumulative record of $57.20 \mathrm{~mm}$.
\end{abstract}

Key words: Coffea arabica L., phenological, flowering stages.

\section{Introducción}

La fenología es el estudio de los eventos periódicos naturales involucrados en la vida de las plantas y comprende el desarrollo, la diferenciación e iniciación de órganos o estructuras y se refiere al estudio de fenómenos biológicos vinculados a ciertos ritmos periódicos como la brotación, la floración, la fructificación, entre otros, y relacionados con el medio ambiente donde ocurren (Schwartz, 1999). Las investigaciones fenológicas se centran en la observación de cambio en la morfología de la planta, con aparición, transformación o desaparición relativamente rápida de determinados órganos o distintos acontecimientos, que se denominan fenómenos fenológicos. El intervalo entre dos acontecimientos o fenómenos fenológicos define un estado

1 Universidad de Oriente, Postgrado en Agricultura Tropical, Núcleo Monagas, Campus Juanico. Maturín, Venezuela.

2 Departamento de Agronomía, Escuela de Ingeniería Agronómica, Universidad de Oriente, Avenida Universidad, Campus Los Guaritos, Núcleo Monagas, Maturín, Venezuela.

* Autor por correspondencia: rsilva@udo.edu.ve

Fecha de Recepción: 28 Octubre, 2014.

Fecha de Aceptación: 17 Diciembre, 2014. 
fenológico o fase de desarrollo de las plantas (Schwartz; 1999; Hack et al., 1992).

La floración es el comienzo de la fase reproductiva del cafeto, los primeros botones florales aparecen generalmente hacia el tercer año de establecimiento del cultivo en el campo, pero alcanzan su plenitud hacia el cuarto o quinto año, este evento comprende varias etapas como la inducción, la iniciación, la diferenciación, el crecimiento y desarrollo, la latencia y la antesis (Barros et al., 1978; Gopal y Vasudeva, 1973; Rena y Maestri, 1984; Wormer y Gituanja, 1970). La floración está asociada estrechamente a las condiciones climáticas de cada región.

La periodicidad del desarrollo floral en café y su relación con los factores ambientales ha sido examinada en varias regiones del mundo como en la India (Gopal y Vasudeva, 1973), en Kenia (Wormer y Gituanja, 1970), en Brasil (Barros y Maestri 1972), y en Colombia (Trojer, 1968). Se ha establecido que en general el fotoperíodo (Franco, 1940), la distribución de los períodos húmedos y secos (Barros et al., 1978; Crisosto et al., 1992; Drinnan y Menzel, 1994) y la temperatura (Mes, 1957) son los principales factores ambientales que la afectan y como resultado las floraciones se concentran o dispersan durante el año. En Colombia (Camayo y Arcila, 1997) y en el este de Kenya por ejemplo, se registran dos períodos principales de floración, que coinciden con el final de períodos secos y comienzo de los lluviosos, no obstante ocurren floraciones esporádicas y de poca magnitud en el año (Alvim, 1960; Wormer y Gituanja, 1970). En Brasil (Camargo, 1985 y Camargo y Camargo, 2001) y Etiopía (Barros y Maestri, 1972; Barros et al., 1978), se registra una sola floración importante en el año.

Más recientemente, un nuevo enfoque se ha dado para la realización de estudios de la floración con el surgimiento de la escala Biologische Bundesanstalt Bundessortenamt and Chemical Industry (BBCH). Esta escala es el resultado de un grupo de trabajo en la República Federal Alemana conformado por el Centro Federal de Investigaciones Biológicas para Agricultura y Silvicultura (BBA), el Instituto Federal de Variedades (BSA), la Asociación Alemana de Agroquímicos (IVA) y el Instituto para Horticultura y Floricultura en Grossbeeren/ Erfurt (IGZ) (Hack et al., 1992).

En la escala $\mathrm{BBCH}$, los estadios principales son 10, iniciándose con la germinación o brotación (estadio 0), según la planta, y finalizando con la muerte o el inicio de la latencia (estadio 9). Al desarrollo vegetativo se le asignan dos macroestadios, correspondientes al desarrollo de las hojas (estadio 1) y de los brotes (estadio 3), este último compartido con el desarrollo de las flores (estadio 5). La floración (estadio 6) y el desarrollo del fruto (estadio 7) completan el código, al crecimiento del brote principal en roseta o de retoños (estadio 2) y al desarrollo de partes vegetativas cosechables (estadio 4) (Hack et al., 1992). Particularmente para el cafeto, Arcila et al. (2002) ampliaron la escala BBCH con la finalidad de describir con detalles las fases de desarrollo de la floración para el cultivo.

Considerando los anteriores planteamientos se realizó este trabajo de investigación con el objetivo de describir las fases fenológicas de floración del café (Coffea arabica L. var. Catuaí Rojo) empleando la escala ampliada BBCH para el municipio Caripe del estado Monagas, Venezuela.

\section{Materiales y Métodos}

\section{Área experimental y material genético}

La investigación se realizó en la Estación Experimental Caripe (INIA-CARIPE), ubicado en la población de San Agustín, municipio Caripe, con $63^{\circ} 32^{\prime} 35^{\prime \prime}$ LO y $10^{\circ} 12^{\prime} 40^{\prime \prime}$ LN, a la altitud de 1.136 $\mathrm{m}$. Los valores promedio de la temperatura oscilan entre 12 y $24^{\circ} \mathrm{C}$ y la precipitación promedio anual es del orden de $1.124 \mathrm{~mm}$. El paisaje característico del municipio es montañoso. La vegetación es bosque húmedo premontano y se destaca por su producción cafetalera; además, en los cultivos de cítricos y hortalizas (MARNR, 1997).

Se utilizó plantas de Coffea arabica L., var. Catuaí Rojo de 10 años de edad, sembradas a 1,0 x $1,5 \mathrm{~m}$, a libre crecimiento bajo condiciones de sombrío, provisto por árboles de bucare (Eryhrina glauca Willd) a un distanciamiento de $14 \times 14 \mathrm{~m}$. En $1 / 2$ hectárea del lote experimental se tomaron en forma aleatoria 30 plantas de café, las que fueron marcadas en el tercio superior con placas de metal enumeradas. En el tercio medio de cada una de ellas, por ser el de mayor producción, se seleccionaron cuatro ramas plagiotrópicas, dos dirigidas en dirección este y las otras dos en dirección oeste, para 120 ramas.

\section{Evaluación de las fases fenológicas}

Las observaciones fenológicas se realizaron entre enero y mayo de 2008. Para identificar la fase 
fenológica de floración se utilizó la descripción de la escala BBCH ampliada propuesta por Arcila et al. (2002), donde se observan las fases de desarrollo fundamentales para el cultivo de café. Las observaciones se realizaron a intervalos de 3 días, en donde se evaluaron cada una de las dos ramas principales en dirección este y oeste, con el fin de determinar los cambios fenológicos en las diferentes etapas del desarrollo floral.

\section{Cuantificación del clima}

La información climática se obtuvo de la Estación Meteorológica ubicada en la Estación Experimental Caripe, perteneciente al Instituto Nacional de Investigaciones Agrícolas (INIA-CARIPE), esta se encuentra a $100 \mathrm{~m}$ del lote experimental, en topografía plana. A partir de los registros diarios de precipitación, temperaturas (máximas, medias y mínimas) y las horas de brillo solar (Insolación), se calcularon valores promedio o acumulados a intervalos de 10 días.

\section{Resultados y Discusión}

\section{Fases fenológicas de la floración}

En la Figura 1 se presenta el desarrollo de los cambios fenológicos de la floración ocurrida durante el período de evaluación en la localidad de San Agustín, municipio Caripe, donde se pudo observar seis estadios fenológicos diferentes, que corresponden a los siguientes códigos: 51 yemas hinchadas, 53 yemas visibles o botones florales indiferenciados, 57 yemas comprimidas o botones florales en desarrollo,
58 botones florales diferenciados o latencia, 59 botones florales en preantesis $\mathrm{y}>60$ botones florales en antesis o apertura floral, descritos ampliamente por la escala BBCH (Hack et al., 1992).

Según la escala BBCH, Hack et al. (1992), el desarrollo de la inflorescencia se inicia cuando en las yemas axilares se observan como hinchamientos en las axilas foliares y termina cuando las flores presentan los pétalos de color blanco pero todavía cerrados. Este proceso puede durar entre cuatro a cinco meses (CENICAFÉ, 2007), en particular para esta localidad la duración de este período fue de aproximadamente 3 meses. El desarrollo de la antesis se inicia con el código 60 que representa las primeras flores abiertas y termina en el estadio 69 cuando el $90 \%$ de las flores están abiertas (Arcila et al., 2002).

\section{Variables climáticas estudiadas}

La diferenciación floral está determinada tanto por factores ambientales como por el fotoperíodo (que puede influir o no), las precipitaciones y la temperatura y por factores internos, los que actúan sobre el crecimiento vegetativo, la floración y el desarrollo de los frutos. Según Camayo et al., 2003; los factores del clima como la precipitación, temperatura e insolación, representan las variables climáticas más relacionadas con el proceso de floración.

\section{Insolación}

Los valores de insolación estuvieron por debajo de 30 horas luz semanales, siendo considerados bajos y por encima de estos se estiman como valores altos

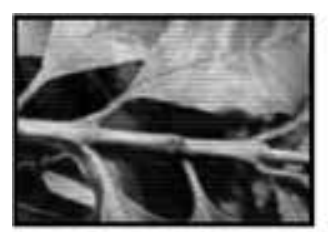

51

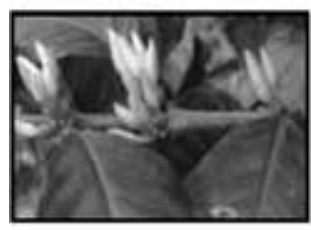

58

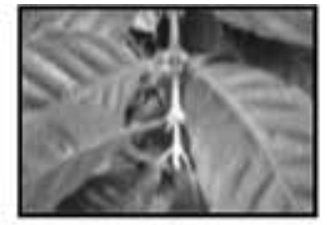

53

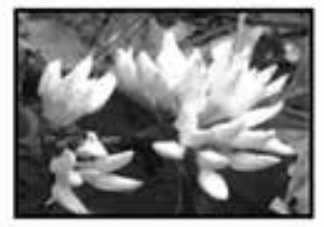

59

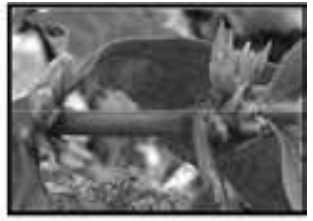

57

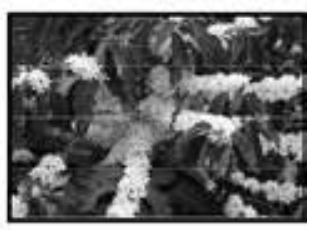

$>60$

Figura 1.Estadios fenológicos del desarrollo de la inflorescencia del café var. Catuaí Rojo, en San Agustín, Caripe, Monagas. 
(Camayo et al., 2003). Basado en esta consideración, durante los seis períodos de evaluación (Figura 1), el factor de insolación fue siempre alto, ya que, según lo expuesto por estos autores, los valores están por encima de 30 por semana, aumentando de forma continua a partir de marzo del 2008 con 5,38 horas diarias de luz en promedio, hasta mayo, con 7,17 horas.

\section{Precipitación pluviométrica}

Los resultados de los registros de lluvia acumulados en enero fueron de $29,6 \mathrm{~mm}$. El valor más alto de precipitación se observa durante febrero, con $83,6 \mathrm{~mm}$, lo que coincide con el menor valor de insolación diaria registrado; sin embargo, para marzo los niveles de lluvia descienden de manera sustancial a $9,40 \mathrm{~mm}$ y es cuando ocurre el mayor estrés hídrico (Figura 1), posteriormente, para abril hay un aumento importante de las precipitaciones con un valor total de $57,20 \mathrm{~mm}$, que finalmente van disminuyendo para mayo con un registro de 43,80 $\mathrm{mm}$. El menor valor registrado de precipitación fue de $9,40 \mathrm{~mm}$.

\section{Temperatura}

Los registros más bajos de temperatura (Figura 1), se observaron durante enero, febrero y marzo, en estos la temperatura máxima no superó los $24,20{ }^{\circ} \mathrm{C}$ y la más baja de las temperaturas se observó durante marzo con $11,82{ }^{\circ} \mathrm{C}$. Los registros de las temperaturas fueron aumentando paulatinamente durante abril y mayo, con valores mínimos de 18,33 y $15,78{ }^{\circ} \mathrm{C}$ y máximas de 24,32 y $26,66^{\circ} \mathrm{C}$, respectivamente.

Los valores registrados de temperatura media, máxima y mínima en marzo fueron los menores con relación a los presentados en los otros meses. Nótese que para mayo hay una coincidencia climática en los bajos niveles de temperatura registrados y de precipitación y un contraste en el registro de los valores de insolación, estos factores están involucrados en las fases fenológicas del fenómeno de la floración.

\section{Inducción de yemas foliares o yemas hinchadas (Estadio 51)}

El primer proceso para que la hoja sea transformada en yema vegetativa reproductiva es la inducción floral. El proceso de inducción (Figura 2A, estadio 51) tuvo una duración de aproximadamente 30 días, ello coincide con lo reportado en trabajos realizados en Colombia (CENICAFÉ, 2007); este proceso puede estar influenciado por un factor específico del ambiente como es el fotoperíodo. Buonafina (1961) advierte que un fotoperíodo crítico estaría en torno a 13-14 horas; sin embargo, este papel del fotoperíodo corto que estimula la inducción floral es cuestionado por Rena y Maestri (1984), quienes señalan que evolutivamente sería difícil imaginar el café como una planta de días cortos en un ambiente en donde el fotoperíodo es prácticamente constante en todo el año, es más probable que sea de días neutro.

De acuerdo con los resultados obtenidos en esta investigación sobre los registros de insolación (Figura 2B), en marzo es cuando ocurre la fase de inducción floral, y es en este mes cuando se presenta aumento de los niveles de insolación de 28,80 horas luz de diferencia respecto del mes anterior, lo que permite ser congruente con lo expresado por Amaral et al. (2006), quienes demostraron que el crecimiento vegetativo no está influenciado por el tiempo de duración del período luminoso, y no causa alteraciones que puedan demostrar el efecto del fotoperíodo acerca de la transición vegetativareproductiva del café, esto nos permite inferir que el fotoperíodo no es transcendente en la floración como sí lo son la precipitación y la temperatura. Asimismo, y confirmando resultados obtenidos en esta investigación, la escasa influencia de la insolación coincide con lo expresado por Rena et al., (2001), cuando manifiestan que durante la inducción floral la acción de las horas luz sobre las hojas no causa un balance hormonal que inhiba el cambio de la planta del estado vegetativo al reproductivo. La planta de café se comporta como una planta de día neutro.

De acuerdo con la ubicación geográfica de la plantación, donde la duración de las horas de luz son menores a 13,7 horas, los efectos reguladores de la insolación sobre la inducción floral carecería de importancia, ello coincide con Cannell (1971) y Alvim (1973), quienes consideran que en regiones cercanas al Ecuador la inducción floral y la floración están reguladas únicamente por la distribución de las lluvias, y mientras más alejado del Ecuador, el cafeto se comporta como una planta de día corto y más importancia tienen las horas de luz en combinación con la distribución de las precipitaciones. 


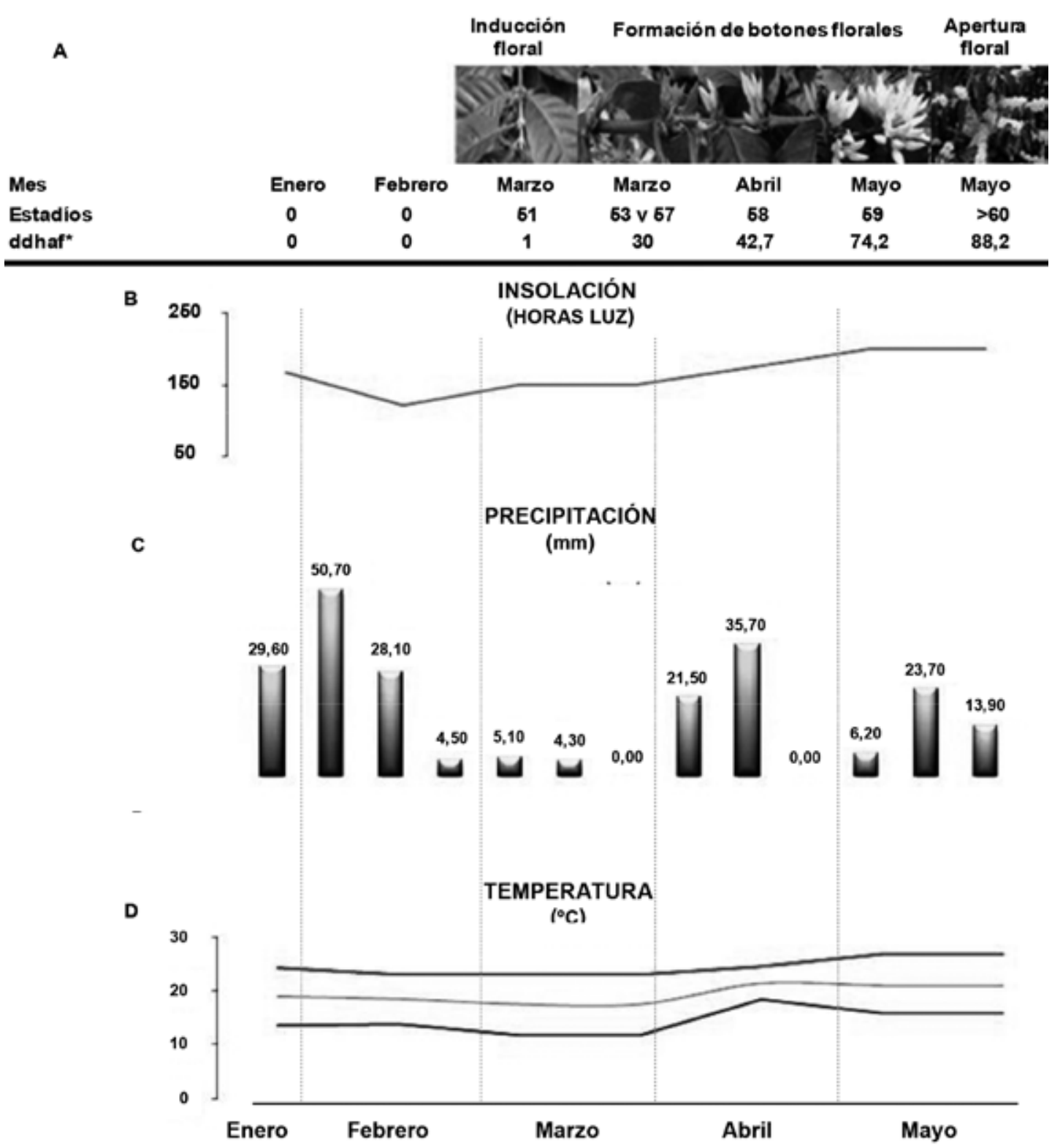

Figura 2. Estadios fenológicos del desarrollo floral del café var. Catuaí Rojo y su relación con las variables climáticas cuantificadas (B) Insolación; (C) Precipitación, y Temperatura (D), en San Agustín, municipio Caripe, Monagas. 2008.

Ddhaf: Días después del hinchamiento de la axila floral

La escasa influencia de la insolación en la inducción floral y el efecto de otros factores climáticos aquí evaluados es manifestado por Barros et al. (1997), cuando exponen que el proceso de inducción floral se inicia debido a una reducción de la temperatura ambiental y la lluvia. Lo anterior es congruente con lo observado en las condiciones de estudio, ya que durante el período de inducción floral (marzo 2008) se registraron los menores valores de precipitaciones, de 9,40 $\mathrm{mm}$, durante el período de evaluación (Figura 2C), lo que estimuló la inducción floral. Por otro lado, Camayo et al. (2003) señalan que los períodos de baja inducción floral parecen coincidir con períodos de brillo solar altos y déficit hídrico; esta contradicción con relación a lo argumentado anteriormente es 
una clara evidencia de que no se debe generalizar la aplicación de conclusiones de estudios acerca del comportamiento fenológico de la floración en distintas zonas cafetaleras.

La influencia de la temperatura en esta etapa fenológica pudiera cuestionarse, toda vez que Mes (1957) y Went (1957) sugieren que las temperaturas día/noche más recomendables para la inducción floral son $27 / 17^{\circ} \mathrm{C}$. Si las temperaturas día/noche están por debajo de $17 / 12^{\circ} \mathrm{C}$ no hay floración, y si están por encima de $30 / 23{ }^{\circ} \mathrm{C}$ se produce mayor número de anormalidades; sin embargo, en esta investigación para el momento de la inducción floral, en marzo, se registraron los más bajos niveles de temperatura mínima de $11,82{ }^{\circ} \mathrm{C}$, temperatura máxima de $22,99{ }^{\circ} \mathrm{C}$ y el promedio de $17,41{ }^{\circ} \mathrm{C}$ (Figura 2D) y ocurrió abundante floración, lo que concuerda con resultados obtenidos por Rayner (1946), donde considera que durante el primer mes de inducción a la diferenciación floral si no se dan las condiciones apropiadas (disminución de la temperatura, desequilibrios bruscos en el balance nutricional, disminución en la concentración de algunas hormonas, etc.) el proceso de diferenciación de las yemas florales es reversible y se desarrollan como yemas vegetativas.

\section{Desarrollo de los botones florales}

Con los resultados obtenidos, durante el período de estudio, se analizó el efecto relativo de los factores climáticos cuantificados respecto de las ocurrencias fenológicas observadas durante el proceso de floración al pasar de un estadio a otro de los botones florales, para ello nos apoyamos en las Figuras 2-A, B, C y D: A partir del momento en que se induce la floración se produce la fase de desarrollo y crecimiento de las yemas florales, en la Figura 2A se presenta la ocurrencia evolutiva de los botones florales (estadios 53, 57,58 y 59), según la escala BBCH (Arcila et al., 2002), la que tuvo una duración de 44 días, estos resultados coinciden con experiencias publicadas por CENICAFÉ (2007), en donde el desarrollo de los botones florales duró 45 días.

\section{Yemas visibles o botones florales indiferenciados (Estadio 53)}

Las yemas axiales no muestran signos de desarrollo de verticilos florales, en la Figura 2A se observa que los primeros botones florales comienzan aparecer a partir de los 30 días después del hinchamiento de la axila floral (fin de marzo), la duración de esta fase fue de aproximadamente 13 días. En este período se registraron las condiciones favorables de insolación, precipitación y temperatura para lograr la diferenciación de los botones florales: déficit hídrico moderado y bajas temperaturas (Figuras $2 \mathrm{C}$ y D). En este trabajo los datos cuantificados acerca de las temperaturas se pudo constatar que durante la fase de desarrollo de las yemas florales la temperatura media fue mayor de $17^{\circ} \mathrm{C}$ y la máxima de $22^{\circ} \mathrm{C}$; sin embargo, la aparición de este estadio ocurrió a los 30 días después del hinchamiento de las axilas florales. En cuanto al efecto de las precipitaciones, los resultados obtenidos concuerdan con los publicados por CENICAFÉ (2007), sobre la ocurrencia de estos estadios al final del estrés hídrico e inicio de precipitaciones moderadas (Figura 2C).

\section{Yemas comprimidas o botones florales en desarrollo (Estadio 57)}

En este estadio los botones florales que se encuentran en crecimiento son de color verde y están cubiertos por una capa delgada de mucílago de color ámbar. En este rango de actividad la mayor presencia de yemas comprimidas o botones florales en desarrollo se detectó en el mismo período cuando ocurrió el estadio 53 (marzo) con una duración aproximada de 10 días, lo que coincide con los datos reportados por Camayo et al. (2003). Los valores registrados de los factores climáticos en este período son iguales a los registrados en el estadio anterior.

En la Figura 2B se observa que para que ocurra el cambio fenológico del estadio 51 a los estadios 53 y 57 se requiere de mayores valores de insolación (horas luz semanales), el acumulado de precipitación es el mismo que cuando ocurrió la inducción de yemas florales y los valores de temperaturas se mantienen prácticamente constantes (Figura $1 \mathrm{C}$ y D), lo que sugiere que debe ocurrir aumento en los registros de insolación y bajas precipitaciones para la ocurrencia de estos estadios fenológicos (53 y 57). Esto coincide con lo reportado por Camayo et al. (2003), quienes asocian la deficiencia hídrica moderada o el brillo solar por debajo de promedios normales (30 hora luz semanal) con la inducción de cambios hacia los estadio intermedios del desarrollo floral, y destacan la importancia de la alta insolación 
para que sucedan los cambios del estadio 51 a los estadios 53 y 57.

\section{Botones florales diferenciados o latencia (Estadio 58)}

En este estadio, los botones florales han alcanzado el tamaño máximo, se encuentran en estado de latencia y son de color verde, según Alvim (1960) y Mes (1957), el período de latencia está controlado por el régimen hídrico que a su vez regula la síntesis de reguladores de crecimiento vegetal. Este estadio ocurrió en la segunda semana de abril (13/04), para ese momento se observan aumentos en los valores de insolación con relación al mes anterior, la precipitación aumentó, alcanzando para abril un acumulado de $57,20 \mathrm{~mm}$, esto sería un nuevo pico de precipitación, y es considerada la variable responsable de la ruptura de la latencia.

Según Crisosto et al. (1992), cuando los botones florales terminan su desarrollo requieren de un efecto acondicionador proporcionado por un período seco o de precipitaciones moderadas, sin este no es posible que los botones florales completen su desarrollo final hasta la antesis. La ausencia de este período seco repercute en que los botones florales permanezcan en latencia o no alcancen el desarrollo hasta la apertura floral. En la presente investigación la ocurrencia de la latencia se manifiesta cuando apenas se inician las precipitaciones durante marzo, lo que coincide con lo reportado por Crisosto et al. (1992). Además, Alvim (1960) sugiere que el período de latencia de los botones florales así como la ruptura del mismo, involucran un mecanismo de reguladores de crecimiento vegetal en donde el ABA y el GA juegan un papel fundamental.

El comportamiento fenológico de pasar del estadio 57 al estadio 58 se podría relacionar con el aumento continuo de los valores de insolación en las semanas previas, estos al final del mes registraron un acumulado de 174,80 horas luz; sin embargo, la ocurrencia de este cambio fenológico está influenciado directamente por el factor precipitación, que va en aumento hasta alcanzar al final de abril un valor de 57,20 mm, lo que coincide con Barros, et al. (1978) quienes evidencian la importancia de las precipitaciones los días previos para la ruptura de la latencia. El incremento de $10 \mathrm{~mm}$ en el régimen hídrico es suficiente para pasar del estadio 58 al estadio 59.
Las variaciones registradas de las temperaturas parecieran sugerir poco efecto en la ocurrencia de estos estadios fenológicos.

\section{Botones florales en preantesis (Estadio 59)}

Los botones florales próximos a abrirse son de color blanco. Según Barros et al. (1978), cuando la latencia se rompe por efecto de las precipitaciones caídas, la yema presenta los pétalos ya formados pero cerrados. Este período duró aproximadamente 14 días y se manifestó a partir de la segunda semana de mayo (14/05) y está asociado a las precipitaciones registradas en el mes anterior, ello sugiere que la lluvia fue suficiente para mantener el suelo húmedo y así lograr el desarrollo de las yemas florales. Según los estudios de Frederico y Maestri (1970) y Gopal y Vasudena (1973), las yemas florales son más grandes y más pesadas en la India que en Brasil y explican este fenómeno, por la mayor disponibilidad de agua en el suelo y la existencia de temperaturas más altas en la India.

\section{Botones florales en antesis o apertura floral (Estadio > 60)}

En este estadio la flor se abre por completo, y la apertura floral normalmente es un proceso rápido y se alcanza el final del proceso de floración. El desarrollo de este estadio se mide según la escala $\mathrm{BBCH}$, por el porcentaje de flores abiertas en el campo (Hack et al., 1992).

A partir del 28 de mayo se inició la antesis, 14 días después de haber ocurrido la latencia, y de allí en adelante se notó gran cantidad de flores abiertas (Figura $2 \mathrm{~A}$ ), esto coincide con Camayo et al. (2003), quienes constataron que la antesis ocurre entre 8 y 14 días después de haberse iniciado las lluvias, lo que es congruente con lo observado en este estudio.

El comportamiento de los factores climáticos cuantificados que afectan al pasar del estadio 59 al estadio $>60$ viene dado por la disminución de las precipitaciones, desde el principio hasta finales de mayo con relación al mes anterior (abril) (Figuras 2 A y C). Las precipitaciones registradas son suficientes para que ocurra el fenómeno de la apertura floral, ya que la cantidad de agua acumulada fue de 43,80 $\mathrm{mm}$ en mayo. Buonafina (1961) señala que en 1946 en Caripe, estado Monagas, $20 \mathrm{~mm}$ de lluvia en cuatro días 
fue suficiente para inducir la floración. Para mayo el factor insolación continúa registrando alzas en sus niveles de horas luz, siendo para el momento de la antesis de 200,60 horas luz, ello influyó en el cambio para el estadio $>60$; así mismo, los registros de temperatura aumentaron. Estos aumentos tanto en la insolación como en las temperaturas máximas, medias y mínimas, sugieren la influencia directa de estos factores climáticos en la formación de las estructuras vegetales observadas en este estadio.

La ocurrencia de la antesis, por supuesto, no es absoluta y sugiere que algunos botones florales se mantienen latentes durante tiempos variables, en espera de condiciones que estimulen el paso hacia la apertura floral, y además es probable que haya latencia en otros estados del desarrollo floral. Generalmente el cafeto florea de dos a tres veces por año, en intervalos variables; sin embargo, se puede determinar el tiempo aproximado de ocurrencia de las distintas etapas fenológicas que ocurren durante todo el proceso de floración.

\section{Conclusiones}

Se identificaron seis estadios fenológicos de la floración: yemas hinchadas (51); yemas visibles o botones florales indiferenciados (53); yemas comprimidas o botones florales en desarrollo (57); botones florales diferenciados o en latencia (58); botones florales en preantesis (59) y botones florales en antesis o apertura floral $(>60)$.

El período desde la aparición del hinchamiento de la axila floral hasta la floración tuvo una duración de 88,2 días.

Se atribuye al estrés hídrico la responsabilidad acerca de la ocurrencia del estadio fenológico de inducción floral. La ruptura de la latencia se debe a las precipitaciones ocurridas al final de abril con un registro acumulado de 57,20 mm.

\section{Literatura Citada}

Alvim, P. de T.

1960. Fisiología del crecimiento y de la floración del cafeto. Café, 2 (6): 54-64.

Alvim, P. de T.

1973. Factors affecting flowering of coffee. In: SRB, A.M. (ed), Plenum Press, New York. Genes, enzymes and population. 193-202 pp.

Amaral, J.A.T.; Rena, A.B.; Amaral, J.F.T.

2006. Crescimento vegetativo sazonal do cafeeiro e sua relação com fotoperiodo, frutificação, resistência estomática e fotossíntese. Pesq. Agropec. 41(3): 377-384.

Arcila-Pulgarín J.; Buhr L.; Bleiholder H.; Hack H.; Meier U.; Wicke H.

2002. Application of the extended BBCH scale for the description of the growth stages of coffee (Coffea spp.). Annals of Applied Biology, 141(1): 19-27.

Barros, S.R.; Maestri, M.

1972. Periodicidade de crecimiento em café. Revista Ceres, 19(106): 424-448.

Barros, S.R.; Maestri, M.; Coons, M.P.

1978. The physiology of flowering in coffee: A review Journal of Coffee Research, 8 (2-3): 29-73.

Barros, S.R.; Mota, J.W.S.; Damatta, F.M. y Maestri, M. 1997. Declive of vegetative growth in Coffea arabica L. in relation to leaf temperature, water potencial and stomatal conductance. Field Crops Research, 54 p.

Buonafina, P.G.

1961. El Café de Caripe. II Jornadas Agronómicas. CaracasVenezuela. Boletín Técnico. 35 p.

Camayo V., G.C. y Arcila P., J.

1997. Desarrollo floral del cafeto en condiciones de la zona cafetera Colombiana (Chinchina-Caldas). Avances Técnicos. CENICAFE 245: 1-8.
Camayo V., G.C.; Chávez C.B.; Arcila P.J.; Jaramillo R.A. 2003. Desarrollo floral del cafeto y su relación con las condiciones climáticas de Chinchiná-Caldas. CENICAFE, 54 (1): 35-49.

Camargo, A. P. 1985. Florescimento e frutificação de café arábica nas diferentes regiões (cafeeiras) do Brasil. Pesq. Agropec., 20(7): 831-839.

Camargo, A.P. y Camargo, M.B.P.

2001. Definição e esquematização das fases fenologicas do cafeeiro arábica nas condições tropicais do Brasil. Bragantia, 60(1): 65-68

Cannell, M.G.R.

1971. Production and distribution of dry matter in trees of Coffea arabica L. in Kenia as affected by seasonal climatic differences and the presence of fruits.Ann. Appl. Biol. 67: 99-120.

CENICAFE.

2007. Sistemas de Producción de café. Publicado en Internethttp:/www.Cenicafe.org.

Crisosto, C.H.; Grantz, D.A.; Meinzer, F.C.

1992. Effects of water deficit on flower open in coffee (Coffea arabica L.). Tree physiology, 10: 127.139.

Drinnan, J.E.; Menzel, C.M.

1994. Synchronization of the anthesis and enhancement of vegetative growth in coffee (Coffea arabica L.) following water stress during floral initiation. Journal of Horticultural Science, 69 (5): 841-849.

Franco, C.M.

1940. Fotoperiodismo em cafeeiro Coffea arabica L. Revista do Instituto do Café, 27: 1586-1542.

Frederico, D.; Maestri, M.

1970. Ciclo de crescimento dos botões florais de café. $C$. arábica L. Revista Ceres, 17 (92): 172-181. 
Gopal, N.H.; Vasudena, N.

1973. Physiological studies of flowering in arabica coffee under south indian conditions. I. growth of flower buds and flowering. Turrialba, 23 (2): 146-153.

Hack, H., H. Bleiholder, 1. Buhr, U. Meier, U. Schnock-Fricke,e. Weber und a. Witzenberger.

1992. Einheitliche codierung der phänologischen entwicklungsstadien mono- und dikotyler pflanzenerweiterte bbch-skala, Allgemein-Nachrichtenbl. Deut. Pflanzenschutzd, 44: 265-270.

\section{M.A.R.N.R.}

1997. Atlas de Estado Monagas. Ministerio del ambiente y de los recursos naturales renovables. Gobernación del estado Monagas. Maturín, Monagas, Venezuela. Pp. 99.

Mes, M.G.

1957. Studies of the flowering of Coffea arabica L. III. Various phenomena associated with the dormancy of the coffee flower buds. Poetugaliae Acta Biologica, 5(1): 25-44.

Rayner, R.W.

1946. Growth and bearing habits of Coffea arabica L. in Kenia and Southern India. East Afric. Agric. J., 11: 251-255.
Rena, A.B.; Maestri, M.

1984. Fisiologia do cafeeiro. In: Simpósio sobre fatores que afetam a produtividade do cafeeiro. Poços de Caldas, Novembro 5-9. Viçosa. Universidade Federal de Viçosa. 2: 1-187.

Rena, A.B.; Barros, R.S.; Maestri, M.

2001. Desenvolvimento reprodutivo do cafeeiro. In; Zambolin, L. Tecnologias de produção de café com qualidade, Viçosa: UFV.101-128 pp.

Schwartz, M.D.

1999. Advancing to full bloom: planning phenological research for the 21st century. 42: 113-118.

Trojer, $\mathrm{H}$.

1968. The phenological equator for coffee planting in Colombia. In: Agroclimatological methods proceeding of the Reading Symposium. Paris, UNESCO. 1. 7: 107-117.

Went, F.W.

1957. The experimental control of plant growth. Ronald Press, New York. 343 p.

Wormer, T.M. \& Gituanja, J.

1970. Seasonal patterns of growth and development of Arabica coffee in Kenia. Part II. Flower Initiation and differentiation in coffee east of Rift Valley. Kenia Cofee, 35: 270-277. 
